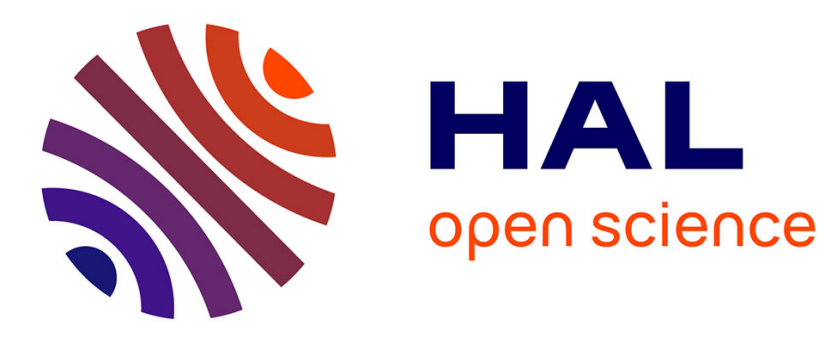

\title{
Du jeune en errance aux zonards
}

\author{
Tristana Pimor
}

\section{To cite this version:}

Tristana Pimor. Du jeune en errance aux zonards. Les Sciences de l'éducation pour l'ère nouvelle: revue internationale, 2014. hal-01228036

\section{HAL Id: hal-01228036 \\ https://hal.science/hal-01228036}

Submitted on 19 Nov 2015

HAL is a multi-disciplinary open access archive for the deposit and dissemination of scientific research documents, whether they are published or not. The documents may come from teaching and research institutions in France or abroad, or from public or private research centers.
L'archive ouverte pluridisciplinaire HAL, est destinée au dépôt et à la diffusion de documents scientifiques de niveau recherche, publiés ou non, émanant des établissements d'enseignement et de recherche français ou étrangers, des laboratoires publics ou privés. 


\title{
Du jeune en errance aux zonards
}

\author{
Tristana Pimor* \\ * Maître de conférences en Sciences de l'éducation, Université Paris Est Créteil.
}

Les Sciences de l'éducation - Pour l'Ère nouvelle, vol. 47, n 1, 2014

Mots-clés : Déviance. Jeunes en errance. Jeunes SDF. Ethnographie collaborative. Trajectoires de vie.

Résumé : L'apparition en France de jeunes en errance, appelés aussi« zonards », suscite des mesures politiques aussi bien sécuritaires que sociales. Néanmoins, peu d'études françaises en sciences humaines se penchent sur ce phénomène. Le flou des définitions et leurs multiples dénominations concourent à développer des analyses ethnocentrées, partielles et à inclure les zonards dans des catégories artificielles sans offrir un aperçu de leurs divers rapports au monde. La littérature Nord américaine, bien que plus éclairante, souffre des mêmes difficultés. Ainsi, par une approche ethnographique impliquée et collaborative avec un groupe de zonards, nous tentons dans cette etude d'appréhender les diverses trajectoires et positions zonardes en nous détachant le plus possible des presupposes normatifs sans pour autant nous contenter d'une simple description subjective.

À partir des années 1990, les espaces urbains français se peuplent de groups de jeunes accompagnés de chiens qui arpentent les rues, mendient (Chobeaux,1996). Cependant dès 1982, le rapport de Gilbert Bonnemaison en faisait déjàétat en soulignant leur dangerosité. À l'époque, la recherche s'intéresse auxjeunes des cités en " galère » (Dubet, 1987). Contrairement à la jeunesse desquartiers populaires dont la territorialisation et la culture hip-hop offrent unecompréhension plus aisée, ces jeunes « en errance » semblent insaisissables. Leurdéfinition, leur appellation constituent un enjeu aussi bien épistémologique,que politique. En fonction de l'approche privilégiée, ils sont considérés commetoxicomanes, précaires ou en difficulté psychologique. En réalité, ces personnescombinent diverses caractéristiques (addictions, vagabondage, délinquance,squat) qui en complexifient la compréhension; ils constituent un groupe socialà part entière puisqu'ils se reconnaissent entre eux et sont catégorisés par les autres groupes sociaux. Ainsi, les premières mesures sécuritaires pour endiguerleur implantation dans les espaces publics sont prises très rapidement (Bonnemaison,1982, p. 56). Nous proposons de revenir sur les définitions scientifiques françaises et nordaméricaines de ces jeunes.Cet examen mettra en évidence des biais méthodologiques, analytiques,que l'approche ethnographique, monographique et inductive, ici d'une duréede quatre années auprès d'un groupe de jeunes qui se nomment eux-mêmesdes « zonards », pourrait amoindrir. Nous démontrerons aussi que ce type detravail ethnographique parait offrir une description plus fine des trajectoires et positions dans la «Zone ${ }^{1}$ ", ce réseau auquel nos enquêtés appartiennent. Lesapproches se référant à l'errance, délivrent un portrait unique " des jeunes enerrance ». Elles seront donc remises en cause afin de développer une

\footnotetext{
${ }^{1}$ Zone : terme employé par tous les zonards pour désigner leur communauté juvénile d'appartenance.
} 
définitionopératoire qui permet de souligner l'hétérogénéité de cette population par lacréation de plusieurs catégories zonardes. Ces constructions permettent d'éviterdeux écueils : celui du regard normatif et d'une analyse trop déterministe, encoretrop présents dans les analyses sur les jeunes de la rue. Des divergences de rapportà la société et à la Zone, liées à des socialisations spécifiques, à des engagementsplus ou moins intenses dans la Zone, ont été repérées. Notre approche permetd'identifier quatre parcours biographiques distincts qui expliquent des engagementset des positions différents dans la Zone.

\section{Jeunes SDF, en errance / Young homeless,throwaways,...}

« À ne pas considérer les SDF comme des acteurs sociaux, à ne pas rechercher lesraisons qui les font croire, agir, se manifester, on s'interdit d'expliquer nombre decomportements et de phénomènes. »(Damon, 2008, pp. 10-11).Les analyses scientifiques des jeunes SDF en France et en Amérique du Nord,auxquels les zonards de notre enquête sont souvent associés, se divisent en

deux grandes voies : l'une plutôt psychologique, l'autre sociologique. Chaqueappellation désignant les jeunes SDF contient en son sein une grille de lectureparticulière et des biais. F. Chobeaux (1996), intervenant social au CEMÉA,est l'auteur de la dénomination " jeunes en errance » servant à identifier leszonards. Les ouvrages usant de cette désignation sont majoritairement le fruitde recherches d'intervenants sociaux. L'utilité de cette terminologie réside danssa capacité à prendre en compte les diverses difficultés que ces jeunes cumulentet à les nommer (Chobeaux, 1996). La description des jeunes en errance faitsomme toute consensus dans ses grandes lignes. En fait, il s'agit bien d'individusâgés de seize à trente ans, accompagnés de chiens, vivant de façon nomade, sansemploi, consommateurs de drogues. Cependant les soubassements analytiquesde la majorité de ces recherches tout comme certaines enquêtes américaines,arguent que l'élection de cette vie marginale et ses pratiques ne peuvent être que

les indicateurs d'une souffrance psychique (Chobeaux, 1996 ; Edelbrock, 1980).« On leur prête des pratiques toxicomaniaques et déviantes dont le paradigmeexplicatif est celui de l'errance vue sous l'angle de la psychologie. » (Bourquetet al, 2004, pp 95). Les errants s'inscrivent ainsi dans un rapport au monde detype ordalique, retraitiste, dû à une éducation déstructurante les déterminant à l'adoption de comportements impulsifs (Chobeaux, 1996).L'utilisation d'autres appellations comme jeunes de la rue, jeunes SDF, younghomeless ou, homeless adolescent, pousse à regrouper dans une même catégoriedes individus qui n'ont rien d'autre en commun que le manque d'habitat légal.Hagan et Mc Carty (1998) s'intéressent ainsi aux jeunes américains qui vivent engroupe dans la rue, fréquentent des foyers d'accueil. Ils constatent que ceuxci afin

de survivre recourent à la prostitution, ce qui pour nous n'a jamais été constaté.Marpsat et Firdion (2001) offrent, quant à eux, une image quantitative de jeunessans domicile reçus dans des lieux d'hébergement, de distribution de repas chaudparisiens. Ils sont décrits comme provenant de familles monoparentales et (ou)d'origine étrangère, parfois en situation clandestine et ne ressemblent pas à nosenquêtés. Le caractère trop général de la dénomination « jeunes SDF » ne permetpas de distinguer les différents cas de figures qu'elle regroupe et donne ainsi uneillusion d'homogénéité des publics jeunes vivant dans la rue (Zeneidi, 2002).La 
multitude des publics touchés par le déficit de logement laisse penser quecette problématique ne revêt ni les mêmes formes, ni les mêmes logiques suivantque l'on soit par exemple une personne en situation irrégulière de séjour, ouencore un travailleur précaire, que l'on soit hébergé chez des amis, en squat,dans son véhicule, en tente, mobile home, etc. Utiliser cette appellation pourles zonards reviendrait donc à les inclure dans une catégorie plus administrativequ'ajustée à la réalité, à leur attribuer des caractéristiques qui ne sont pas les leurset en réduisant la complexité des populations de la rue, à rendre encore moinslisibles les trajectoires. Par ailleurs, en France, qui dit jeunes SDF dit déliaison,exclusion. Pour Guillou (1998), des cumuls de ruptures familiales, sociales,scolaires rendant difficile l'adaptation des jeunes les plus démunis aux injonctionséconomiques expliqueraient leur condition de rue. Cette fragilisation des liensfamiliaux qui déracine le jeune serait due à l'évolution « postmoderne » de notre

société moins encadrante, moins structurante, dans laquelle les individus sontsommés de trouver seuls leur place (Le Rest, 2006). Cette lecture évacue doncd'un revers de main des facteurs subjectifs pourtant importants. Outre-Atlantique,des études ethnographiques se centrent sur les liens entre pratiques délinquanteset facteurs familiaux, scolaires, situationnels suivant un modèle quelquepeu déterministe (Hagan \& Mc Carty, 1998). L'imprégnation quotidienne dansun contexte violent et pauvre génèrerait automatiquement des comportementsdéviants relevant d'une culture de classe sans décrire le processus à l'oeuvre. Lesconclusions semblent alors identiques : la précarité familiale, la maltraitanceconfortée par la pauvreté, la déviance parentale conduisent les jeunes à fuir leurfoyer et à s'affilier avec des pairs déviants faute de mieux.Pour les jeunes nommés : runaways, throuaway, street youth, youth at risks, lescauses identifiées sont essentiellement familiales. Un modèle éducatif désadapté,des conduites parentales déviantes, inadaptées concourent par imitation audéveloppement de comportements déviants qui sont perçus comme ordinairespar l'enfant (Whitbeck et al., 1997).Pour la catégorie itinérant, traveller, nomadic people of the street, les descriptionss'attachent davantage à la façon de vivre de ces jeunes. Ces approches,auxquelles nous nous apparentons, mettent en exergue la fonction socialisatricemarginale de la rue, réfutant ainsi le caractère désorganisé des conduites de cesjeunes (Parazelli, 2002). En effet, un lien est souvent tissé entre les comportements déviants de ces jeunes et une existence vue comme anomique. $\mathrm{Au}$ mieux,les déviances de ces jeunes de rue sont interprétées sous un jour plus rationnel,comme des adaptations aux conditions de vie de rue. Les pratiques déviantes nepeuvent se limiter à cette fonction car elles sont aussi créatrices d'identité, de lienaffectif et révélatrices d'un mode de vie, "d'une culture " propre à la catégorie desjeunes vagabonds étudiée (Finkelstein, 2005).Cependant, bien qu'une distinction entre différentes jeunesses de la rue sedessine grâce à l'usage de l'ethnographie, chaque étude aborde sa populationcomme un ensemble homogène. Les disparités de parcours et les divers positionnementsdans leur espace de « jeunes SDF » ne sont pas évoqués. Les choixméthodologiques privilégiant des observations non impliquées, sur une brèvedurée, auprès d'individus sans cesse changeants, n'autorisent pas à atteindre laprofondeur des trajectoires. Pour contrer cette difficulté, une approche ethnographiqueimmergée "d'une famille » de jeunes de rue nous a semblé pouvoirapporter de nouvelles données permettant de complexifier et de rendre plus compréhensibles les trajectoires de nos enquêtés. Notre recherche s'est ainsicentrée sur une jeunesse de rue : "les zonards » et non sur tous les types dejeunes SDF existants. Bien évidemment la monographie souffre d'un manquede représentativité qui empêche toute velléité de généralisation non mesurée etnon rigoureuse. 
Cependant, la finesse du sens émique qu'offre l'ethnographiedévoile des logiques inaccessibles par l'emploi d'outils plus extérieurs et autorisede nouveaux questionnements sur le phénomène. Notre approche se distinguedes travaux antérieurs par son regard plus intérieur d'un univers de la rue, parsa volonté de ne pas considérer les acteurs comme des agents passifs, ni hors desjeux sociaux. Pour ce faire, nous avons décider de porter une attention aussi bienaux socialisations passées de ces jeunes qu'à leur carrière déviante, leur socialisationzonarde (Lahire, 2001 ; Becker, 1985 ; Berger, Luckmann, 2008). Cetteanalyse tente de prendre en compte aussi bien des influences liées à l'appartenancesociale, à la famille, à la scolarité que celles relatives à l'engagementdans une vie déviante. H.S. Becker souligne que les carrières déviantes sontprécédées de vécus excluants, sans pour autant les détailler (1985). Nous avonsdonc voulu identifier si dans les trajectoires des jeunes zonards des vécus, destransmissions antérieures favorisaient des engagements différents dans la carrièrezonarde. Cependant, nous n'avons pas voulu nous borner à repérer dans ceuxciuniquement des ruptures mais avons tenté d'élargir aux inclinaisons culturelles,éducatives, écologiques, scolaires qui y concourraient. En ce sens, la convocationdu concept de socialisation plurielle de B. Lahire nous a permis de mesurer lesinfluences de trois sphères de socialisation : la famille, l'école et les pairs (2006).Néanmoins, nous estimons que pour qu'elles s'opérationnalisent en accord avecle mode de vie déviant zonard, un engagement subjectif, un étiquetage socialet l'apprentissage de pratiques d'une culture déviante zonarde sont nécessaires(Becker, 1985). Notre cadre théorique est donc particulier puisqu'il tente deréunir deux approches objectives et subjectives. Nous utilisons les concepts desocialisation, en tenant compte des inculcations et des constructions de la réalité,et d'expériences sociales, de carrière plus interactionniste et donc moins déterministe (Lahire, 2001 ; Dubet, 1994 ; Becker, 1985 ; Berger \& Luckmann, 2006).Ce cadre, à cheval entre un certain constructivisme et l'interactionnisme, s'estdessiné durant la phase de terrain et découle des données construites durantl'observation.

\section{Une ethnographie coproduite entre chercheur et enquêtés}

Ma rencontre avec la Zone et des zonards débute en 2007. Au cours d'un travaild'initiation à la recherche sur les conduites à risque toxicomaniaques mené dansune structure CAARUD 2, je m'entretiens avec quatre jeunes que les éducateursont repéré comme ayant des pratiques risquées (échange de seringue, relationssexuelles non protégées). Nia, l'un d'entre eux me propose alors son aide pourde futurs travaux de recherche. Il deviendra mon premier informateur. Nia est âgé de vingt-huit ans et vit dans la rue depuis huit ans. Il a quitté le Jura,sa compagne, suite à des déboires amoureux, et ne supportait plus son travaild'ouvrier à la chaine. Issu d'une famille dont les parents ont divorcé, il a été élevéprincipalement par ses grands-parents paternels, des agriculteurs jurassiens. Sonpère, policier, qui avait obtenu sa garde et celle de sa soeur, ne s'est pas occupéd'eux. Afin que je réalise de nouveaux entretiens sur la thématique des conduitesà risque, Nia me présente un an après Benoît. Il a vingt-huit ans et vit dans lemême squat que lui. Benoit vient d'une famille dont le père travaille dans lebâtiment et la mère est femme au foyer. Ils résident en zone rurale. Ses parentsn'ont pas de diplôme et Benoit a arrêté ses études avant l'obtention d'un CAP.En conflit avec eux du fait de son mode de vie déviant (drogue, free parties),Benoit a décidé de partir à ses vingt-et-un ans. Je rencontre un an avant, dans lemême cadre que Nia, Clara, une jeune fille de dix-neuf 
ans, qui a vécu depuisa petite enfance en foyer de l'enfance, et Passe-Muraille, un jeune homme dedix-sept ans. Clara s'est installée dans la rue, il y a un an, après avoir déjà vécude manière intermittente en squat et en tente depuis ses seize ans. À quatorzeans, Passe-Muraille fugue de chez ses parents, ouvriers sans diplôme, en raisonde désaccords «politiques » (son père est engagé au front national et lui se revendiqueanarchiste). Éduqué à la dure, il a vécu son enfance dans une petite villede Charente.

\subsection{Enquêteur, enquêtés, un apprivoisement mutuel en terredélinquante}

Je m'aperçois que ces jeunes que je voyais mendier dans les rues de Bordeaux,partagent une vision du monde commune, des références culturelles spécifiques.Nia, suite à une conversation lors de laquelle je lui expliquais mon désir de mieuxcomprendre leur mode de vie, me propose de venir au squat réaliser des observations.Effrayée par des représentations stéréotypées de la vie en squat et de seshabitants, je décline l'offre. Je crains de me retrouver face à un groupe d'individus totalement shootés, dans un lieu lugubre et sale. Les entretiens ne suffisant pas àélaborer de descriptions précises de leur mode de vie, l'observation participantes'est alors imposée. Pour retrouver Nia, je me poste devant le supermarché où ilmendie régulièrement. Il arrive avec quatre amis, acquiesce à ma demande maispréfère au préalable, parler du projet d'étude à ses co-squatteurs : «la Family » 3 .Il me donne son adresse, m'y convie la semaine suivante. Le squat se situe enbanlieue de Bordeaux, dans une ville occupée majoritairement par des habitantsde classes moyenne et supérieure. Je pense trouver un immeuble insalubre oùdes individus drogués s'entassent sur des matelas crasseux à même le sol. Je suisface à une maison mitoyenne taguée. De vieux vélos, de la ferraille sont entassésdans un coin de la cour. Je frappe à la porte, Brest, l'un des squatteurs âgés devingt ans, vivant d'ordinaire en camion, vient m'ouvrir. Le chauffage fonctionne,le squat est rangé, assez propre. Les murs sont tagués de phrases : " la violenceest pure et naturelle, la société l'a rendue impure » 4, de paroles de Léo Ferré : «l'anarchie, c'est l'ordre sans le pouvoir », de plaisanteries, de noms de gens, detous les chiens (Diesel, Boulette, Mesrine...).Au cours des quatre années de recherche, je rencontre une quarantaine depersonnes. Je réalise une observation participante d'une année découpée en deuxpériodes de six mois entre lesquelles je continue à côtoyer les jeunes. Durant lespremières semaines, mes craintes liées à une potentielle agression me conduisentà suivre Nia partout où il se rend : dans la rue, les magasins, les associations, chezdes amis en appartement. Au bout de quatre mois, je m'autorise à rester sans luiau squat et commence à suivre d'autres habitants comme Poly et Kundevitch, uncouple de jeunes âgés respectivement de 19 et 22 ans. Poly, fille d'un patron depetite entreprise et d'une mère de milieu bourgeois, réside pour partie chez sesparents et au squat. Son père peu diplômé est devenu chef d'entreprise à la sueurde son front et grâce au réseau social de son épouse. Ils résident dans une stationbalnéaire côtée. Poly, atteinte d'amblyopie, vit trois jours par semaine chez sesparents et le reste du temps au squat dans lequel néanmoins, elle ne s'installerajamais. Kundevitch a, quant à lui, vécu dans un pavillon de banlieue de classemoyenne avec son père, représentant en peinture et sa mère secrétaire comptable.Sa mère, issue d'un milieu relativement populaire, a obtenu son baccalauréatcontrairement à son père non diplômé. Suite au divorce parental, Kundevitch,agent en maintenance en système incendie, décide de prendre un appartementtandis que son petit frère de huit ans son cadet s'installe avec sa mère. Kundevitch 
démissionne quelque temps après, perd son appartement, se retrouve chezsa grandmère puis dans la rue.En parallèle, je mène dix-huit récits de vie auprès des squatteurs et certainsde leurs amis en débutant par Nia qui a déjà participé à deux entretiens. C'estau cours de celui-ci que j'apprends que les habitants me suspectent d'être unpolicier infiltré ou un "indic' ». Il faudra attendre l'incarcération de Yogui, leleader du groupe âgé de vingt-cinq ans, réputé pour sa violence guerrière, pourqu'une vraie confiance se tisse. Je lui écris, je soutiens moralement sa compagne Mumu restée au squat. Son père est infirmier, sa mère aide-soignante. Mumu avingt ans, ses parents ont divorcés ; elle ne se sent ni bien chez eux, ni totalementà son aise dans le squat. Elle alterne entre ces deux modes d'habiter, suivant lesconflits qui se nouent.Entre les deux périodes d'observation, je garde des contacts avec Yogui et $\mathrm{Nia}$;nous travaillons régulièrement sur les analyses en cours. Nia partant en cure desevrage, Yogui devient mon second informateur. Il lit mes écrits, m'interrogesur les notions et concepts, acquiesce à mes constats et mes hypothèses. Nia estmoins impliqué ; il s'est réinséré et aspire à une vie ordinaire lorsque je reprendsl'observation. Après plus de trois années à m'épauler, autant dans la mise enrelation avec des zonards que dans la critique de mes conclusions, il s'en désintéresse.D'autres m'interrogent régulièrement sur mes notes, mes théorisations.Ils lisent mon carnet de bord, le commentent. Comme Kundevitch, vivantdans la rue depuis quatre ans qui me déclare : " tu pourras dire qu'on est desbons gens ». " La Family » se réunit tous les soirs autour du repas unique de lajournée, partage tout. Chacun contribue de différentes manières aux besoinscommunautaires (aides sociales, deal, vol...). Ces pratiques sont communes àla Zone. Chaque week-end donne lieu à une fête dans un squat ou en Freeparty. Au squat, autour d'un feu, une dizaine de personnes rassemblées avec leschiens discutent, boivent des bières, fument des joints pendant que la musiquetourne doucement par respect pour les voisins. Les uns préparent le barbecue,d'autres prennent des drogues plus fortes de manière discrète. Les discussionsvont bon train. Des conflits peuvent éclater allant jusqu'au règlement de compte.La soirée se termine au petit matin en regardant le soleil se lever. Le paradoxedes comportements contemplatifs, solidaires, anarchistes et ceux plus agressifsexigent une totale décentration pour en saisir le sens. S'affiliant aux Travellersmais aussi héritiers des Punks, ils évoquent une idéologie libertaire et contestentle fonctionnement de notre société actuelle : coercitive, conformiste, individualiste,mercantile, inégalitaire et aliénante. L'étrangère, que je suis, doit alors opterpar peur de mésinterprétation pour un travail collaboratif avec les enquêtés, puisse distancier grâce à l'écriture et l'analyse (Clifford \& Marcus, 1989). Ce choixde travail avec les enquêtés a, tout comme la méthode ethnographique, été dictépar la nature méfiante des zonards envers les individus extérieurs au groupe. Dufait des activités délinquantes commises et de trahisons expérimentées dès leurenfance 5, les zonards sont sur leur garde. Cette monographie collaborative m'adonc paru convenir aux contraintes de ce terrain et de son objectif : comprendreles trajectoires zonardes. Elle permet, de par la proximité qu'elle induit, d'explorerle quotidien de la Family de manière plus compréhensive, plus profonde, comptetenu de ma posture qui se veut en partie subjectiviste. La démultiplication desgroupes observés n'aurait pas permis d'obtenir autant de détails sur la vie desacteurs : d'une part, une certaine méfiance aurait pu s'installer du fait de conflitslarvés éventuels voire de rivalités entre les « familles » 6 de rue, d'autre part, untemps conséquent d'apprivoisement est nécessaire à l'acceptation du chercheur.C'est donc au bout d'un an que ma place d'écrivaine du squat émerge pour neplus évoluer. 


\subsection{De l'errant au zonard}

C'est à l'occasion d'un entretien avec Benoit que le problème de la définitionzonarde apparaît en prenant la forme d'une lutte lexicale : « je m'intéresse aux jeunes en errance, à la façon dont ils vivent... ».« Les jeunes quoi ? J'ai pas compris » (Benoît). Je me rallie à un vocabulaire plus proche :

« Aux jeunes de la rue ».

Taraudée par cet échange, je questionne Nia sur les termes qu'ils utilisent.« Nous, c'est zonards ou traceurs ».Je lui fais part de l'utilisation «d'errant » par les travailleurs sociaux. Interrogatif,il me lance :« Hein ? Quoi ? Hérons ?» (Nia).Ces mots semblent alors, pour lui, vides de sens. Ils ne lui correspondent pas.Il me fallut du temps avant d'accepter l'obligation du recours au terrain, de la

définition opératoire et d'oser écarter le paradigme de l'errance. Ce paradigmeconsidère que pour expliquer la trajectoire des jeunes dits « en errance », il fautavant tout porter son attention sur la conduite errante vue comme un symptômede difficultés psychiques liées à une éducation familiale carencée, maltraitante oufaite de ruptures. Pour traduire cet état, l'attribution d'un nom extérieur, doncobjectif comme « jeunes en errance » se révèle efficace.Or, mes premières conclusions, issues d'une analyse thématique de sept entretiensrévèlent une vision du monde propre aux zonards qui ne peut se réduire àun simple symptôme. Tous sont animés de projets de voyage : G Grâce au cametar 7, je vais tracer de ville en ville et de chaque paysapprendre la manière dont ils fabriquent leurs bijoux et les revendredans les marchés » (Clara),d'un rejet affiché pour la vie normée : "Ma liberté c'est tout ce que je demande, après, tout ce qui est tune et

tout ça j'en ai rien à foutre, j'ai pas envie de travailler pour payer un

loyer » (Passe-Muraille).Ils affirment haut et fort que la rue est « Un choix ! Moi, je veux la rue c'est tout »,qu'ils désirent vivre en relation avec la nature : On va se poser sûrement dans les bois et quitte à vivre comme unhippie : c'est-à-dire à cultiver, chasser » (Passe-Muraille),revenir aux sources, à une vie communautaire, voire tribale. D’emblée les valeurssemblent claires et conscientisées : Valeurs... : déjà fraternité, respect, no jugement, no vol, entraide $\gg(\mathrm{Nia})$.

Les termes « jeunes en errance », SDF, ne peuvent recouvrir cette réalité. " Laconsidération du point de vue des jeunes est capitale dans le développementde la connaissance du phénomène » (Hurtubise et al, 2000, p. 183). De fait, lanomination et la définition de la population repérée doivent émaner du terrain." Les définitions utiles et utilisables doivent ici organiser un travail de fixationsémantique qui est de l'ordre de l'indication opératoire, en ce sens que le motdéfini y devient un index, c'est-à-dire qu'une telle indication est plus généralequ'un nom propre mais moins générique qu'un concept universel. » (Passeron,2006, pp. 270). Suite à cette réflexion, j'interroge les participants sur le nomqu'ils estiment leur correspondre et l'adopte. Zonard reste le plus répandu.

\section{La pluralité zonarde : des trajectoires biographiques qui agissent sur les carrières zonardes}

En collaboration avec La Family, à partir de catégorisations indigènes qui ne sontpas nommées dans leur réalité, ont été dégagées quatre catégories permettant demesurer et de discerner les formes d'engagement des individus dans la culturezonarde et les diverses séquences de carrière ainsi que leurs places dans la Zone :Traveller, Zonard 
Experts, Zonard Intermittent, Satellite (Becker, 1985). Cettecarrière débute par la séquence satellite dans laquelle certains s'arrêtent, d'autrespoursuivent jusqu'à la seconde séquence de zonard intermittent, stoppent oucontinuent vers la troisième, de zonard expert, et enfin passent ou non à laquatrième, celle de Traveller, qui se situe presque en fin de carrière.Modèle de la carrière zonarde et de ses séquences :

1. L'entrée : Satellite

2. Le tâtonnement et l'apprentissage : Zonards intermittents

3. L'engagement et l'aboutissement : Zonards experts

4. L'aboutissement / sortie : Travellers

Pour repérer des constantes des acteurs positionnés dans l'une ou l'autre descatégories, toutes les données relatives aux socialisations passées (type d'éducation,inculcations déviantes ou conformes aux normes légitimes) et auxexpériences (déclassement, mobilité sociale) prenant place aussi bien dans lafamille, l'école, que dans les groupes de pairs ainsi que celles plus écologiques(type d'habitat parental, quartier) ont été extraites des observations, des entretiens

et des récits de vie. De la même manière les professions des parents, leursniveaux d'études ont été répertoriés. Des tableaux, permettant de mesurer lareprésentativité des items les plus synthétiques pour chaque catégorie de zonards,ont été effectués. Concernant les expériences sociales, une analyse thématique des récits de vie apermis de saisir que certaines d'entre elles ont favorisé l'inclinaison vers une viezonarde plus ou moins engagée et donc vers le statut associé. Ces catégorisationssont évidemment plus caricaturales, plus cohérentes que ce qu'elles sont dans la

réalité de la Zone. Néanmoins, elles permettent de saisir les fonctionnementssousjacents à l'adhésion à cet univers. L'appellation de la catégorie « Traveller »est issue du langage indigène. Les autres, non nommées initialement par leszonards, ont été créées en collaboration avec la «Family ».

\subsection{Satellites : attirance pour la déviance zonarde et la réussite sociale légitime}

Les Satellites correspondent aux individus sédentaires qui gravitent autour desZonards et qui n'adhèrent pas aux pratiques sous-consommatrices, anarchistes.Ces membres de la Zone fréquentent les Free parties, des squats lors de soirées oude visites amicales, mais ne veulent pas s'y établir. Ils vivent principalement chezleurs parents ou en appartement.Pour ceux qui resteront dans cette séquence, la valeur travail, le goût pour lesbiens de consommations et l'importance d'un habitat légal les différencient ets'expliquent par des expériences sociales dans les univers familial et scolaire relativementpacifiés. Sur le plan familial, les divorces fréquents semblent avoir étéabordés par les jeunes de manière sereine. Les difficultés économiques inhérentesà la monoparentalité ont été résolues grâce à l'activité professionnelle des mèresqui se sont données sans compter. La méritocratie professionnelle colore doncles transmissions que ces jeunes ont reçues de leur mère et les engagent à persévérerdans la recherche d'emploi ou dans l'aboutissement de leur formation.De capitaux économiques disparates, ces familles se situent dans des milieuxmoyens et supérieurs. Toutefois, il ne va pas pour autant de soi qu'elles aident

matériellement leurs enfants qui, dès qu'ils ont quitté le giron familial, doiventse débrouiller seul.Au collège et au lycée, bien qu'ils ne soient pas des élèves ni en difficulté,ni très investis, une remobilisation prenant place en classe de première, aprèsune période de désinvestissement, leur permet d'obtenir un baccalauréat. 
L'expériencescolaire est décrite comme un temps d'amusement avec les camarades,une activité impliquant un certain travail qui lorsqu'il est fourni, se voit récompensé.La méritocratie scolaire est donc bien présente dans les représentationsdes Satellites et s'étend même à l'univers du travail. La bifurcation vers la Zones'effectue durant une période de démobilisation scolaire (souvent en classe detroisième et de seconde) où la socialité avec les pairs prend le pas sur l'apprentissage. À ce moment là, les Satellites autorisés à sortir le samedi soir rencontrentles Free Parties et les zonards qui les fréquentent. La teuf 8 est donc la première

marche d'accès à la Zone. Ils apprennent à reconnaître les différents styles demusique techno alternative (acid core, hard tech...), l'histoire de la Spirale Tribe- premier sound system arrivé en France suite à la répression des Free Partiespar Margaret Thatcher - qui fonde le mythe des Travellers et à consommer desdrogues. Pour être un Satellite, il faut être « Teufeur », c'est-à-dire amateur deFree Parties et partager un certain état d'esprit : être libre, ouvert aux autres,tolérant, capable de fonctionner en communauté, respecter la nature, ne passe rendre en teuf uniquement pour se droguer ou vendre des stupéfiants. Cemode de pensée s'acquiert par la fréquentation d'un petit groupe d'adeptes.La musique en dehors de sa fonction première hédoniste est aussi un outil desociabilité pour les zonards, un liant entre les participants à ces fêtes (Racine,2002). Les danseurs se trouvent plongés hors du temps, dans une fête qui peutdurer plusieurs jours. Libérés des contraintes sociales, ils ressentent une sorte decommunion avec les autres qui est accentuée par les prises de drogues hallucinogènes.Ici la drogue est lien. Chez les satellites peu engagés encore dans la culturezonarde, ces Zones festives servent aussi d'exutoire à des frustrations générées parle monde du travail, de la formation (Vaudrin, 2004). La performativité de laposition Satellite requiert par ailleurs un sentiment de bien-être, d'appartenanceainsi que la maîtrise, le respect de règles qui encadrent certaines pratiques. Ils'opère par exemple une distinction ente mauvaises et bonnes drogues qu'il fautrespecter. L'héroïne est vectrice de vices du fait de la dépendance physique qu'ellegénère, ses adeptes sont « des camés ». À l'opposé, la cocaïne, les hallucinogènessont considérés comme des médias de la sociabilité zonarde et de la connaissance

de soi. De même, les usages de drogues sont réglementés. Ainsi, les conduitesextrêmes non maîtrisées sont condamnées. Le partage des psychotropes constitueune norme. Si les Satellites s'inscrivent dans la Zone, ils ne sont qu'à sa lisière.Ils utilisent juste les principes qui les intéressent, de manière instable dans letemps, et paradoxale avec certaines de leurs valeurs, et ne renoncent pas à unevie ordinaire.

\subsection{Les zonards intermittents : du vilain petit canard à l'indécis}

Les Zonards Intermittents qui stagnent sur cette seconde marche de la carrièrezonarde, se caractérisent par leur hésitation à se convertir à la Zone et par uneprédominance du sexe féminin. La dureté du mode de vie et le difficile renoncementà la valeur travail, transmise par des mères fortement engagées dansl'emploi, expliquent le difficile passage à l'étape Zonard Expert. Les familles deces jeunes appartiennent aux classes moyennes mais connaissent néanmoins desdifficultés financières. Les contenus des socialisations sont en relatif accord avecla norme, valorisent la réussite sociale et scolaire, expliquant qu'ils jouissent decapitaux scolaires plus importants que les Zonards Experts. Même si l'absentéismecaractérise les dernières années de scolarisation au collège, la sortie dusystème scolaire en classe de terminale ou après le 
baccalauréat, s'expliqueuniquement par le désir de vivre dans la rue. Bien que l'école ne les ait pas aidéà faire face à des accidents biographiques (viol, décès, apparition d'un handicap,dépression), elle n'est en effet jamais remise en cause en tant qu'institution. Cesaccidents biographiques constituent l'un des tournants biographiques expliquantl'attirance de ces jeunes pour la Zone. L'indifférence de la famille et de l'écoleface à ces difficultés génère un sentiment de négation de leur problématiqueet par extension de leur individualité. Entre 16 ans et 18 ans, la fréquentationde Free Parties, grâce à des pairs qui les initient, permet la rencontre avec deszonards et déclenche une prise de conscience. Les acteurs s'aperçoivent que leursproblèmes niés par les adultes de l'école et de la famille sont considérés, écoutéspar ces jeunes zonards. L'attitude de la famille et de l'école, incapables d'offrirune reconnaissance et une écoute à leurs souffrances, facilite alors l'engagementdes Zonards Intermittents. La Zone, qui accepte la diversité et qui favorise lesrelations affectives, leur offre un étayage, une reconnaissance positive.

Cette séquence Zonard Intermittent est une étape d'hésitation pour tous,y compris pour ceux qui accèdent aux stades suivants, et un tremplin vers unmieux-être à venir. À cette période, les tensions entre les jeunes et leur familles'accroissent et entraînent des mises à la porte temporaires, des fugues sans queles liens ne soient pour autant rompus. Les allers-retours entre rue et famille

se routinisent. Les relations qu'ils entretiennent avec la Zone comme avec leur famille oscillent entre fusion et rejet. Leur engagement dans l'univers de la rue estdonc intermittent : le cadre de référence zonard et celui légitime inculqué par lafamille et l'école s'entrechoquent. La socialisation secondaire zonarde impliquealors un fort ancrage affectif pour perdurer et des protocoles pédagogiques spécifiques.Ainsi, les petits amis pour les filles et les «pères de rue » qui sont desZonards experts servant de modèles et d'enseignants pour les garçons, c'est-à-dire

des pourvoyeurs d'orientation, leur enseignent les techniques d'obtention d'argent,de nourriture, de biens matériels, de consommation d'opiacé, d'ouverture etde gestion d'un squat, de rationalisation ainsi que les règles de vie dans cettecommunauté (Berger \& Luckmann, 2008). Ces nouvelles pratiques zonardess'additionnent à celles acquises au cours de la séquence Satellites et participentde leur intégration plus engagée dans la Zone. Le passage du stade Satellites austade Zonards Intermittents s'organise au travers des consommations d'opiacésdevenues plus régulières et de la dépendance physique qu'elles créent. Il semble,en effet, que bien que mal perçue, il s'impose à tous vrais zonards d'avoir aumoins testé suffisamment l'héroïne pour la critiquer. Les Satellites rencontrésl'ont aussi essayée mais ils ne l'utilisent que sporadiquement, "en descente detrip ». Fonctionnant comme une part d'un rite d'initiation, la prise d'héroïneassigne au nouvel adepte la qualité d'initié aux drogues et lui offre une meilleure

reconnaissance dans la Zone. Durant cette expérience, le novice est instruit parun expert qui lui prépare un trait ou une seringue d'héroïne et lui évoque lessensations qu'il va ressentir. L'héroïne est en définitive, considérée comme "Ladrogue », celle qui requiert, de par ses propriétés addictives, un engagement toutentier de l'individu. Cette pratique démontre la loyauté, l'obédience du noviceà la vie zonarde, prouve l'adoption d'un positionnement déviant. Le zonard

intermittent ne peut plus trahir les siens, il est lui-même pris dans cette dépendancepsychotropique et dans une transgression de la loi qui peut lui valoir unecondamnation judiciaire. L'héroïne occupe ainsi au sein du groupe une fonctionde marqueur d'appartenance et de lien. Les zonards intermittents partagentavec les experts les mêmes expériences d'exploration de leur subjectivité, dela souffrance 
corporelle. Ce marquage dans le corps se poursuit aussi par destatouages, des piercings, des écarteurs, des scarifications qui signent visuellementleur appartenance.

D'autres pratiques comme la mendicité, la vie en squat, les demandes d'aideset d'accompagnement sociaux appris durant cette séquence, socialisent lesacteurs au fonctionnement zonard, leur accordent une place en son sein et lesengagent indirectement par l'étiquetage social qu'elles impliquent. La consommationd'héroïne pousse en effet les utilisateurs à recourir aux offres du secteuren addictologie, le manque de revenus à solliciter le travail social et à mendier.

Perçus comme des «punks à chien » ou « des jeunes en errance » par les riverains,les intervenants sociaux, cette désignation déviante les confine au rôle d'outsiderzonard et prend le pas sur tous les autres rôles sociaux qu'ils pourraient occuper(Becker, 1985). Ne désirant pas s'en délivrer, du fait des bénéfices affectifs qu'ilsretirent de leur appartenance à une famille de rue, ils acceptent alors cette identitésociale qui les engage davantage dans la Zone. Cette désignation contraint leurspossibilités d'interactions tant dans leurs formes que dans leurs destinataires.

Aux yeux de la société, ils sont devenus des zonards qui ne peuvent interagiravec les hors-zones que sur le modèle de la demande d'aide, de l'opposition et demanière symétrique qu'avec des zonards.L'apprentissage de l'idéologie zonarde, étrangement ressemblante à la penséeanarcho-primitiviste qu'ils ne connaissent pourtant pas, engage aussi ces jeunes.Le choix de ne pas impacter sur l'environnement par une surconsommation tientà ce choix politique. Pour autant, les zonards intermittents ne parviennent pasencore à adhérer à toutes les valeurs et surtout à la révolte que cette critiquesociale contient. La valeur travail n'est pas vraiment remise en cause, le fonctionnementdémocratique non plus. Ils aspirent à une société plus égalitaire, plussolidaire, plus communautaire mais sans vouloir détruire notre fonctionnementsocial actuel. Pour passer à la séquence suivante de zonard expert, la socialisationzonarde doit parvenir à démanteler les socialisations primaires et familiales(Berger \& Luckmann, 2008). Pour ceux dont les expériences sociales dansces deux sphères se sont révélées pourvoyeuses de « rage» et dont les socialisationsprimaires contenaient déjà des principes proches de ceux de la Zone, laconversion à la socialisation zonarde est aisée (Dubet, 1987). Pour les autres, lessocialisations primaires prennent le pas et incitent, au fil du temps, les acteurs àretourner vers un mode de vie conventionnel.

\subsection{Trajectoire d'experts : réaction aux inégalités}

La catégorie des Zonards Experts correspond donc à l'aboutissement de lacarrière zonarde et regroupe des individus ayant des trajectoires biographiques etun rapport au monde voisins. Ces zonards vivent à temps plein dans des squats, défendent des idéaux mêlant anarchisme et tribalisme. Ils sont très engagésdans la vie de rue et maitrisent, enseignent aux nouveaux zonards des pratiquespropres à ce mode de vie (mendicité, deal, consommation de stupéfiants, langagespécifique et abrupt, bagarre), les règles qui les encadrent, les valeurs qui lessous-tendent (hédonisme, solidarité, honnêteté, aventure, rencontre de l'altérité)leur permettant d'accéder à une place reconnue dans la Zone.Sur le plan des biographies infantiles et adolescentes, des points communsont été identifiés chez les acteurs qui parviennent à cette séquence et qui yrestent. Les familles des zonards experts appartiennent aux milieux populaireset précarisés. Les parents sont ouvriers, employés, ont connu des périodes dechômage ou encore plus rarement tirent leurs revenus d'activités délinquantes(vols, trafics de 
stupéfiants). Une lutte pour subvenir aux besoins familiauxrythme leur quotidien. Toutefois, en secteur rural, la solidarité encore présentedans ces milieux et au sein des familles élargies, leur permet, malgré les difficultéséconomiques, d'adoucir leurs conditions de vie. Les repas familiaux, entreamis, les fêtes, les matchs de rugby, de football sont ainsi évoqués comme desévènements récurrents et joyeux de leur enfance, ce qui les engage à vouloirmaintenir certaines valeurs et pratiques de leurs socialisations familiale et declasse (Lahire, 2001). Les contenus populaires de la socialisation familiale soumisà ce contexte, enjoignent à concevoir le temps de manière présentiste, hédonisteet s'accordent avec ceux de la socialisation zonarde (Hoggart, 1970). La toléranceaux consommations d'alcool, de drogues, aux bagarres entre jeunes, synonymesd'apprentissage de la virilité, d'hédonisme se retrouve dans le cadre de référencesde zonards en devenant des pratiques valorisées. L'organisation même de la

famille au sein de laquelle le père jouit d'une place de patriarche est reprise parles zonards experts. Yogui occupe ainsi une place de leader dans la Family, définiepar une autorité en partie traditionnelle, jamais remise en cause. Sur le planéducatif, le modèle dominant est relativement coercitif, voire perçu de l'extérieurcomme erratique. Le châtiment corporel employé comme technique depunition produit une vision naturalisante de la violence que l'on retrouve dans laculture zonarde. Le respect de la hiérarchie familiale, de la politesse, de la réussitescolaire (mesurée à l'obtention de la moyenne permettant le passage dans laclasse supérieure) et l'accomplissement des tâches domestiques attribuées constituentles domaines dans lesquels les transgressions sont les plus sanctionnées. Enrevanche, les sorties tardives entre copains, les consommations de psychotropes,les bagarres, bref tout ce qui se déroule entre pairs, n'est pas soumis à un contrôleparental stricte. Ces activités font partie du processus de maturation permettantle passage à l'âge adulte.Du fait de leur situation sociale et de leurs attitudes éducatives repéréescomme dysfonctionnelles (trop rigides ou erratiques), les services sociaux interviennentdans ses familles et ce faisant participent, par leur simple présence, àune stigmatisation précoce. À cette désignation de « familles à problèmes » s'additionnentcelle du chômage des parents et celle relevant de leur appartenance àdes territoires dépréciés socialement. L'environnement dans lequel les familles résident n'est pas sans conséquence sur le rapport au monde développé parles Zonards experts. Leurs lieux d'habitation se situent dans des zones rurales,périurbaines stigmatisées - définies par le sens commun comme regroupant des « dégénérés »-, où les problèmes de délinquance et de chômage sont présents,et côtoient, pour la campagne médocaine dont sont issus la majeure partie deces jeunes, la richesse d'exploitants viticoles fortunés. Ces acteurs, enfants deparents sans emploi, ouvriers agricoles ou dans le bâtiment, baignent donc dansun climat particulier de ségrégation où la présence de viticulteurs très favorisésaccentue leur sentiment de relégation. La mixité sociale n'est donc pas effectivecar ces deux milieux (ouvriers agricoles, propriétaires d'exploitations) ne semêlent que rarement, si ce n'est au sein des établissements scolaires où là encoredes classes de niveaux reproduisent la séparation entre l'univers des pauvres et desindividus aisés. Cette situation provoque donc un sentiment certain d'injusticesociale qui s'additionne à celui expérimenté dans le quotidien hors l'école. L'étiquetagenégatif de la famille, les inégalités sociales dont les futurs zonards ontprécocement conscience, concourent à l'élaboration d'un rapport oppositionnelau monde légitime, favorable à une inscription dans un mode de vie déviant. Lesentiment de domination pousse les jeunes à penser que l'affiliation à un premiergroupe de pairs déviants sera plus porteur qu'un investissement scolaire dont lesdés sont déjà pipés. La croyance en la méritocratie sociétale et scolaire s'est vueen 
effet ébranlée par les injustices professionnelles rencontrées par leurs parents et par celles qu'ils ont eux-mêmes vécues au collège.Yogui : "Et de là est montée un peu ma rage. Le fait que mon père quiest un gars bien, rentré dans la société, se soit fait enculer par la société,alors qu'il faisait tout pour élever ses enfants le mieux possible ».De surcroît, le regard porté par l'école sur les dysfonctionnements familiaux etleurs signalements auprès des services de l'aide à l'enfance, délégitiment l'institutionscolaire qui devient complice aux yeux de ces jeunes de la stigmatisationet du contrôle social centrés sur les familles en situation de précarité économique.

L'école devient une arène catalysant la disqualification sociale familiale.Une forte loyauté familiale, due à la nécessaire protection de ses membres sanscesse outragés par l'extérieur, accentue la logique de réaction au stigmate sous laforme d'une rébellion à la culture scolaire. Celle-ci se traduit par des absencesfréquentes, des comportements violents, insolents, confortés par des orientationsvers des filières de relégation qui entraînent ainsi l'arrêt des études entre quatorzeet seize ans. Non détachés de la valeur travail, importante dans leur culturefamiliale malgré les vécus parentaux douloureux, les zonards experts tentent detrouver des emplois. Ces expériences se soldent par la confirmation que le mondedu travail s'inscrit dans l'exploitation des plus faibles par les plus forts. Ainsi lesexpériences vécues dans les sphères scolaire et professionnelle confortent leurinterprétation d'un monde basé sur la domination et concourent à développerleur désir de vivre autrement, de prendre une place différente de celle vers laquelleleur appartenance sociale les oriente.Résidant, comme évoqué, dans des lieux où la délinquance est présente, cesjeunes ont d'une part, bénéficié d'opportunités, se sont socialisés avec des pairsinscrits dans des conduites déviantes et d'autres part, ont cherché à trouver uneplace sociale autre que celle à laquelle ils étaient destinés en s'affiliant à un groupeà même de les valoriser (Cohen, 1955). Ils testent ainsi divers groupes déviants : « racailles », « resdkin » dans lesquels ils continuent d'apprendre certainespratiques délinquantes (bagarre, deal, vol, consommation de drogue). Toutefois,ces groupes ne répondent pas à leurs attentes. Les « racailles », trop matérialistes,inscrites dans la société de consommation, ne sont pas assez opposées aufonctionnement social qu'ils décrient ; les « Redskins », trop « bourgeois », necomprennent pas réellement les enjeux du milieu dont ils sont issus. Au même

moment, les acteurs se distancient de leurs familles, se rendent en Free Parties oùils rencontrent des Zonards. Fascinés par l'ambiance, ils se rapprochent de leurfutur groupe d'appartenance.Trash : «En fait à 12, 13 ans j'ai commencé à fuguer, quoi ! [...]. Enfin de compte j'ai commencé à faire mes premières teufs, à découvrirce que c'était des camions, voilà, les chiens, tout ça. Je kiffais trop, j'aitrop kiffé, quoi ».Ils alternent tout d'abord vie en squat et chez des copains, dans la famille, puiss'installent dans la rue. Ce temps de latence permet aux jeunes d'apprendre le« système D 9 », d'acquérir des connaissances sur la consommation de stupéfiants,la violence physique, les valeurs (solidarité, honnêteté, sous-consommation,hédonisme, présentisme), les normes et l'idéologie (anarchiste, tribaliste)présentes dans la Zone. Cet apprentissage dure en moyenne un an et est supervisépar un aîné « un père de rue ».Enquêtrice : « T'as un plus âgé qui t'a un peu coaché au départ ? »Trash : " Ouais Yogui il m'a connu j'étais minot, j'avais 15, 16 ans.

Ouais carrément après j'avais pas que lui. J'en ai eu plein des pèresde rue [...] ».Puis, en voyageant seul, les garçons néophytes s'affranchissent de leur père derue, deviennent à leur tout le « père de rue » d'un autre, accèdent au statut dezonard expert. C'est par la gestion, la participation à une ouverture d'un squat,le détachement de sa première famille de rue et la création d'une nouvelle famillede rue que l'acteur se voit 
reconnu comme zonard expert. Il doit par ailleurs êtreporteur de l'idéologie zonarde, pouvoir la transmettre, faire respecter les règlesqui encadrent les pratiques. Ainsi, les conflits physiques s'inscrivent toujoursdans une logique de sanction envers un individu transgresseur et ne doiventpas être gratuits. L'activité de deal, quant à elle, doit répondre à un impératifqualitatif en terme de produit et ne doit pas viser à s'enrichir. Si elle est pratiquéeà une échelle jugée importante, elle doit alors soutenir un projet particulier,comme celui de s'acheter un camion. Ainsi cette pratique, tout comme celle dela mendicité, n'est pas uniquement mobilisée pour répondre à des conditions devie difficiles et subies mais constitue une alternative à la vie conventionnelle etentre autre, au travail précaire auquel les acteurs auraient été contraints. Ce sontdes moyens de se lier, d'éprouver du plaisir pour la consommation de drogue, derefuser la course au temps à laquelle les travailleurs sont soumis. Ces pratiquessont donc des révélateurs d'un désir de faire société autrement, des emblêmesd'une posture politique. Elles ne relèvent pas exclusivement de transmissionsliées à des socialisations passées, d'une rationalité économique mais aussi d'unelogique éthique que les expériences sociales antérieures et la carrière zonarde ontéveillée.

\subsection{Traveller : un Zonard Expert pas comme les autres}

Cette catégorie correspond à l'aboutissement idéalisé de la carrière zonarde et sesitue à la frontière de la sortie de carrière.Julie : «Là si je pouvais avoir la vie que je voulais... plus tard, là

prendre quelques années pour voyager, pour... vivre dans moncamion, vendre mes bijoux ».Les travellers s'identifient à leur habitat : un camion ; au mouvement : ilsvoyagent plus que les autres. Ils sillonnent le monde, au gré des emploissaisonniers, des festivals, des approvisionnements de drogues. Ils stationnentdans les bois, les champs en été, aux abords de squats en hiver. Du fait de leurmobilité, les Travellers sont moins dépendants du fonctionnement de la Zonebien qu'ils partagent les mêmes valeurs. Cette indépendance s'explique d'unepart, par le peu de temps passé en squat, en position de Zonard Expert, et parleurs capitaux familiaux économiques plus favorables et culturels plus légitimes,mais néanmoins critiques. Les vécus infantiles, familiaux ne laissent pas transparaîtred'animosité envers une société jugée injuste, régie par la logique dela domination sociale. Les expériences sociales scolaires, au sein de la sociétéglobale sont apaisées et n'engendrent pas de révolte particulière. Cependant, lapolitisation des familles leur offre, dès le lycée, une lecture critique du fonctionnementsocial. La scolarité de ces jeunes se déroule sans accrocs jusqu'au baccalauréat.Leurs parents, souvent de profession intermédiaire, les ont encouragésà poursuivre des études post Bac pour certains. Ils trouvent, pour les autres,rapidement des emplois après l'obtention du baccalauréat. Toutefois, cesexpériences professionnelles et universitaires jugées peu épanouissantes, lesconduisent à remettre en cause leur rapport au monde et aux normes légitimes.Humiliés, exploités, robotisés dans l'univers professionnel, perdus, non-aidés,pris dans une compétitivité inhumaine à l'université, ces jeunes jugent que notresociété, sous couvert d'offrir à tous les mêmes chances, participe à l'aliénation desmoins dotés, exerce en catimini un tri social. Remettant en cause les logiques decompétition, de rentabilité, d'enrichissement qui fondent pour eux les universprofessionnel et universitaire, ils bifurquent et cherchent un autre mode de vieque celui, conventionnel, dans lequel ils se projetaient. Au moment où ces interrogationsprennent place, les acteurs se rendent en Free Parties et s'inscriventtour à tour dans toutes les séquences précédentes de la 
carrière zonardes. Leuradhésion à la zone répond, tout comme chez les zonards experts, à une logiqueéthique révélée par les expériences post-bac peu gratifiantes. Ils acquièrent selonle même modèle que les zonards experts, les pratiques, l'idéologie, les normeset valeurs de la Zone. Néanmoins, la période de vie en squat est plus courte, laprise de distance avec le "père de rue » plus rapide car l'acquisition du véhiculeest plus aisée. Ils bénéficient d'aide de la famille ou sont plus en mesure, au vu deleur niveau d'études, d'accéder à un emploi contracté uniquement dans ce but.Shanana, âgée de vingt-six ans, dont les parents possèdent un restaurant, travailleainsi durant 6 mois dans un magasin de vêtements avant d'acheter avec soncompagnon un camion. Pour les zonards experts, l'achat d'un camion nécessitebeaucoup plus d'efforts du fait de leur rapport révolté au monde et de capitaux

moins à même de le leur permettre. Nombreux sont ceux qui n'y parviennent pasou qui doivent attendre longtemps. Plus âgés que les autres types (25 ans), arrivésplus tardivement dans la Zone (après 19 ans), ils aspirent au calme et se situentdans un rapport au monde moins oppositionnel. L'objectif ne consiste pas icià affronter la société comme pour les Zonard Experts mais à bâtir une manièrede vivre alternative dans laquelle ils se sentiront épanouis. Leur engagementpolitique se tourne donc davantage vers la construction, la proposition que versl'affrontement.

\section{La définition par terrain, la co-construction d'idéauxtypes zonards : une fenêtre sur une autre réalité}

L'approche développée dans cette recherche a permis, d'une part, une lecturedu phénomène zonard détachée des analyses le considérant comme le simplereflet d'acteurs passifs désoeuvrés, et d'autre part de mettre en avant les différentspositionnements dans la zone. L'organisation zonarde bien que discontinue n'enest pas moins cohérente. Les degrés d'engagement dans la culture de la Zone, liésaux socialisations et expériences sociales familiales, scolaires, aux interactions quifacilitent l'inscription plus ou moins impliquée dans la carrière zonarde, ont puêtre identifiés en se référant aux catégories co-élaborés avec les enquêtés. Cettelecture autorise alors la prise en compte des facteurs passés en tant qu'influençantle futur rapport au monde des acteurs dans une perspective diachronique maisaussi synchronique. Pour beaucoup, issus de quartiers de relégation, ils auraientpu adhérer à un autre groupe déviant. Ainsi l'élection de la voie zonarde est aussiun choix dans un panel d'orientations disponibles et ne relève pas de déterminismessociaux implacables. Les interactions au quotidien doivent aussi êtreprises en compte. Les labellisations déviantes concourent à la performativité del'identité zonarde attribuée. L'ethnographie impliquée et collaborative, permet,l'espace d'un temps, d'expérimenter la condition des enquêtés, de se décentrerde sa propre vision des choses par obligation situationnelle et interactionnelle,et autorise ainsi une compréhension plus sensible, plus proche de ce qu'est lavie zonarde de l'intérieur : une organisation complexe avec des engagementsdivergents, un hédonisme gouverneur malgré sa dureté, une culture qui s'inspired'ancrages populaires et non une existence passive et misérable.

\section{Bibliographie}

Becker H. S. Outsiders. Paris : Métailié, 1985.

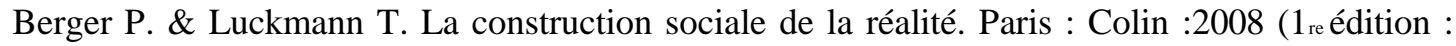
1966).

Bonnemaison G. Face à la délinquance : prévention, répression, solidarité :

rapport au Premier Ministre, 1982. En ligne : [www.ladocumentationfrancaise.

fr/docfra/rapport_telechargement/var/storage/rapports-publics/834037801/0000.pdf ], consulté le $11 / 05 / 2012$.

Chobeaux F. Les nomades du vide. Paris : La Découverte, 1996.

Cohen A. K. Delinquent Boys. The Culture of the Gang. New York : Free Press, 1955.

Damon J. La question SDF. Paris : PUF, 2008.

Dubet F. La galère. Paris : Fayard, 1987. 
Dubet F. Sociologie de l'expérience. Paris : Seuil, 1994.

Edelbrock C. Running away from Home : Incidence and Correlates amongchildren and youth referred to mental heatlh services. Journal of familial issues,1980, vol. I., pp. 210-228.

Finkelstein M. With no direction homes : homeless youth on the road and in thestreets. Wadsworth, Case studies on contemporary social issues, 2005.

Goffman E. Stigmate : Les usages sociaux des handicaps. Paris : Minuit, 1975.

Guillou J. Les jeunes sans domicile fixe et la rue ou « Au bout d'être énervé ». Paris :L'Harmattan, 1998.

Hagan J. \& Mc Carthy B. Means Street, Youth crime and homelessness.Cambridge : University Press, 1998.

Hoggart R. La culture du pauvre. Paris : Minuit, 1970.

Hurtubise. R, Vatz Laaroussi. M \& Dubuc. S. Jeunes de la rue et famille. Desproductions sociales et des stratégies collectives au travers des mouvances du réseau.Université de Sherbrook, rapport de recherche présenté au Conseil Québécoisde la Recherche Sociale, 2000.

Lahire B. L'homme pluriel : les ressorts de l'action. Paris : Hachette Littteratures,2001.

Le Rest P. L'errance des jeunes adultes, causes, effets, perspectives. Paris : L'Harmattan,2006.

Marpsat M. \& Firdion J.-M. Les ressources des jeunes sans domiciles et ensituation précaire. Recherches et prévisions, 2001, $\mathrm{n}^{\circ}$ 65. pp. 91-112.

Passeron J.-C. Le Raisonnement sociologique. L'espace non-poppérien du raisonnementnaturel. Paris : Michel, 2006.

Parazelli P. La rue Attractive, Parcours et pratiques identitaires des jeunes de la rue.Québec : Presse Universitaire de l'Université du Québec, 2002.

Whitbeck L. B, Hoyt D. R. \& Akley K. A. Abusive family backgrounds andlater victimization among runaway and homeless adolescents. Journal ofresearch on adolescence, 1997, Vol VII, n ${ }^{\circ} 4$. pp. 375-392.

EN 47-1.indd 91 17/03/2014 17:38 